\title{
Caracterización macroscópica y microscópica de lana ovina en el litoral sur de Perú
}

\author{
Montesinos, I.S. ${ }^{@} ;$ Catachura, A. ${ }^{2}$; Perezgrovas, R.G. ${ }^{3}$; Fioravanti, M.C.S. ${ }^{1}$ y Sereno, J.R.B. ${ }^{4}$
}

'Programa de Post-Grado en Ciencia Animal. Escuela de Veterinaria y Zootecnia. Universidad Federal de Goiás. Goiânia (GO). Brasil. ${ }^{2}$ Escuela de Veterinaria y Zootecnia. Universidad Nacional Jorge Basadre Grohmann. Tacna. Perú.

${ }_{3}^{3}$ Instituto de Estudios Indígenas. Universidad Autónoma de Chiapas. San Cristóbal de Las Casas (Chiapas). México.

${ }^{4}$ Empresa Brasilera de Pesquisa Agropecuaria. Embrapa Sede. Brasilia (DF). Brasil.

\section{PALABRAS CLAVE ADICIONALES}

Kemp.

Mestizaje.

Calidad de fibra.

Rebaños criollos.

\section{RESUMEN}

En Perú, la producción de lana se posicionó en mejor lugar para competir con las fibras sintéticas, debido al precio oscilante del petróleo a nivel mundial. Este estudio objetivó caracterizar lana ovina, según cualidades macroscópicas y microscópicas de interés etnológico y económico. En el municipio de lte, litoral sur de Perú, fueron muestreados mechones de lana en 20 ovejas criollas de los Humedales, 20 criollas de las chácaras y seis mestizas Hampshire Down. También fue medido el peso vivo de los ovinos. Los resultados de las características físicas de la lana y pesos vivos fueron transcritos a planillas electrónicas, para analizar mediante estadística descriptiva usando el programa computacional $R$ y crear un histograma. Las ovejas mestizas y criollas de las chacras eran más pesadas que las criollas de los Humedales. En los tres grupos predominaron las fibras cortas, con ventaja de los ovinos criollos por menor presencia de kemp. Los criollos de los Humedales poseían mayor largura de mechón y rendimiento al post desengrase. Los diámetros de fibra de las ovejas criollas de lte indicaron una lana entrefina y confortable. Estos rebaños poseen fibras útiles en la confección textil industrial, pero el grupo criollo de los Humedales puede ser seleccionado por sus mechones más largos para uso artesanal. En las chacras existen cruzamientos absorbentes de hembras criollas con mestizos Hampshire Down. Es urgente crear núcleos de ovinos locales buscando su conservación y fomento entre los criadores, quienes deben organizarse y pedir apoyo gubernamental para la confección textil, logrando una ovinocultura sustentable en lte.

\section{Macroscopic and microscopic characterization of sheep wool in southern coast of Peru}

\section{SUMMARY}

\section{ADDITIONAL KEYWORDS}

Creole flocks.

Crossbreeding.

Fiber quality.

Kemp.

INFORMATION

Cronología del artículo.

Recibido/Received: 08.10.2016

Aceptado/Accepted: 22.05.2018

On-line: 15.07 .2018

Correspondencia a los autores/Contact e-mail:

ivansm.vet@gmail.com
In Peru, wool production positioned in better place to compete with synthetic fibers, due to oscillating oil prices around the world. This study aimed to characterize sheep wool, according to macroscopic and microscopic qualities of ethnological and economic interest. In Ite's municipality, southern coast of Peru, wool fleeces were sampled in 20 creole sheep from Humedales, 20 creole sheep from farms and six Hampshire Down crossbreds. Also was measured the sheep live weight. The results of physical characteristics of wool and live weights were transcribed into electronic spreadsheets, to analyze through descriptive statistics using the software $\mathrm{R}$ and create a histogram. The crossbred and creole sheep from farms were heavier than creole sheep from Humedales. In the three groups predominated the short fibers, with advantage of the creole sheep by minor presence of kemp. Creole sheep from Humedales had greater staple length and yield after scouring. The fiber diameters of Ite's creole sheep indicated an entrefine and comfortable wool. These herds have useful fibers in industrial textile confection, but the creole group from Humedales can be selected by their longer fleeces for artisanal use. On farms exist absorbent crosses of creole females with Hampshire Down crossbreds. It is urgent to create nucleus of local sheep looking for their conservation and promotion among the breeders, who must organize themselves and require governmental support for the textile confection, achieving a sustainable sheep farming in lte.

\section{INTRODUCCIÓN}

En Perú, el uso de lana está relacionado al diámetro de fibra, siendo dos tercios de la producción utilizado en ropa, en cuanto un tercio en la manufactura de colchas y tapetes (Tinoco, 2009). La producción peruana de lana en los últimos años no mostró variación 
significativa, siendo entre 10 a 13 mil toneladas (TN), reportándose en 2004 la cantidad de 11,6 mil (Díaz, 2007) y en 2012 bajó levemente para 10,9 mil TN (Díaz, 2013). Desde la década de 1980 hubo una disminución en la producción mundial de lana, debido a factores del mercado internacional, como el inicio de la comercialización de substitutos sintéticos y otras fibras naturales (algodón) a precios competitivos, así como el gran abastecimiento australiano de lana, afectando a países productores de fibra (Viana, 2008). Este fenómeno provocó pérdida de interés por la producción lanífera de ovinocultores, técnicos e investigadores (Aliaga, 2009). Para Díaz (2007) en Perú los precios de la lana también fueron afectados, sumando a eso la desorganización de los productores, la valorización de la carne ovina y el comercio basado en lana de baja calidad con fibras gruesas (> 30 micras), sabiendo que la preferencia de la moda son tejidos leves de fibras finas y superfinas. Todo esto hizo con que rebaños productores de lana disminuyesen y los restantes fuesen cruzados con razas de carne, perdiendo calidad en la fibra.

Desde 2005 las fibras sintéticas derivadas del petróleo están elevando sus precios, ya que el precio del barril oscila en su valor, sujeto a las volatilidades propias de su naturaleza como recurso no renovable y susceptibilidad a las disputas geopolíticas en el Oriente Medio. Estas tendencias en el precio de los substitutos, además de una importante corriente ecológica entre consumidores, han revitalizado el uso de las fibras naturales, posicionando la lana en mejores condiciones, para competir en el consumo de fibras textiles (Tinoco, 2009). Algo reflejado en las exportaciones peruanas de lana, que están creciendo lentamente en el ramo de bolas top, generando ingresos de 1,7 millones de dólares americanos, siendo el destino Italia, Alemania, China, Ecuador, Irán, Japón, Suiza y Reino Unido (Díaz, 2013). Según Amarilho-Silveira et al. (2015), la China es el principal comprador mundial de lana, incrementando sus adquisiciones por la mejora en la economía de los Estados Unidos y Europa, dos de los mercados que más adquieren productos hechos con ese material. En 2017 el Perú exportó unos 6, 7 millones de kilos de lana sin cardar, a 2,8 dólares americanos el kilo (Agrodata Perú, 2018).

Coincidentemente, en varios países de América Latina y Europa está siendo estudiada la calidad de lana de las ovejas criollas (Rojas et al., 2005), para establecer relaciones genéticas que confirmen su evolución histórica, así como registrar características físicas importantes para la confección textil industrial y artesanal (Parés et al. 2007a). Siendo una alternativa de explotación, ya que en el futuro posiblemente las razas especializadas entren en decadencia, debido a mudanzas climáticas que afectan los insumos con los cuales son alimentadas. Estas razas ovinas locales al poseer mayor adaptación a diferentes ambientes y condiciones de manejo (Fulcrand, 2005), serían la mejor opción, para tornar las crianzas sustentables con menos inversión económica. Ese abordaje motivaría la recuperación y conservación de los ovinos criollos, la identidad de sus propietarios, así como sistemas tradicionales de producción, los cuales son cada vez más escasos. Algo que ocurre en el municipio de Ite, donde existen dos tipos marcados de ovinocultura, según ambiente y tipo de criador, siendo ambos dirigidos para la producción de carne. En el ecosistema de los Humedales se encuentran 19 familias Aymara, las cuales pastorean ancestralmente rebaños de pequeños rumiantes, con un número de 4234 ovinos según el último reporte. Ya en las chacras están los agricultores familiares, que en su mayoría crían ovinos junto a bovinos y caprinos bajo el sistema de estaca, siendo registrados 126 criadores y 1879 ejemplares (MDI, 2014a; Montesinos et al., 2015).

Debido a la carencia de información en varios aspectos sobre estos pequeños rumiantes en esa región de Tacna, el presente trabajo objetivó describir macroscópica y microscópicamente la fibra de lana de ovejas criollas y mestizas Hampshire Down, caracterizándolas etnológicamente y determinando la calidad lanera de estos animales, buscando así otras fuentes de renta económica y la conservación de los rebaños criollos para una ovinocultura sustentable en Ite.

\section{MATERIAL Y MÉTODOS}

En el municipio de Ite, región Tacna, litoral sur de Perú (Latitud sur 17 $50^{\prime} 27^{\prime \prime}$ y Longitud oeste 70 57'47'), fueron colectados mechones de lana de ovejas adultas, a partir de los dos años de edad o cuatro dientes incisivos inferiores permanentes, en 20 hembras criollas "cara blanca" de los Humedales (CRIHU), 20 criollas "cara blanca" de las chacras (CRICH) y seis mestizas "cara negra" Hampshire Down (MHD), siendo dos de los Humedales y cuatro de las chacras (Figura 1). Estas ovejas se escogieron aleatoriamente en siete criaderos de los Humedales y 18 criaderos de las chacras, representando un número significativo de muestreo comparado al total de rebaños y ovinos en Ite, sabiendo que la población criolla ha disminuido por cruzamientos absorbentes con MHD.

El biotipo más común de oveja criolla era la variedad llamada chuña por los pastores Aymara, que poseía

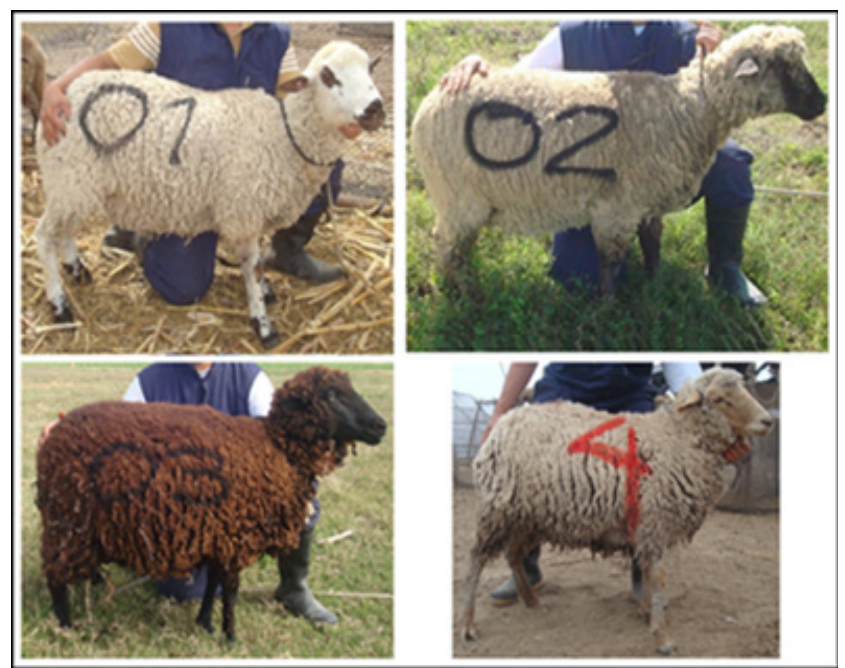

Figura 1. Oveja criolla "cara blanca" (01) y mestiza Hampshire Down "cara negra" (02) de los grupos muestreados. Ovejas criollas negra (03) y castaña (4) en riesgo de extinción (Creole sheep "white face" $(01)$ and Hampshire Down crossbred "black face" (02) from sampled groups. Black (03) and brown (4) creole sheep in risk of extinction). 
cara y lana blanca, con manchas típicas de color negro o marrón en el rostro y patas, parecidas a sus ancestros Churros de España. Las ovejas mestizas tenían lana blanca o algo amarilla y cara negra o gris, según mayor grado de hibridación con $\mathrm{HD}$, que estaría relacionado al rostro negro de esa raza inglesa de carne, muy popular en la región, con núcleos de animales puros en la sierra de Tacna (Montesinos et al., 2015). Fueron encontrados algunos ejemplares criollos de lana y cara tanto negra como castaña clara, pero en cantidad ínfima, lo que indicaría riesgo de extinción de esos dos fenotipos peculiares, situación que también comentaron Stemmer et al. (2017), pues por la presión del mercado de fibra blanca esos recursos zoogenéticos pueden desaparecer. Esta heterogeneidad de colores fue descrita en el Borrego mexicano Chiapas (Perezgrovas \& Castro, 2000), el ovino Navajo-Churro de Estados Unidos (Sponenberg \& Taylor, 2009), ovinos chilenos Chilote (De la Barra et al., 2011) y la oveja argentina Formosa (Galdámez et al., 2012), evidenciando la biodiversidad del ovino criollo en América.

En Ite, los tres grupos muestreados fueron formados según la información oral de los criadores, principal herramienta para separar los ovinos, ya que la mayoría de productores sabía el origen e historia evolutiva de sus rebaños, conocimientos también registrados por Peña et al. (2015) en ovinocultores argentinos. Los productores participantes de la investigación determinaban cuál oveja era criolla o cruzada según la coloración del rostro y biotipo del animal, criterio empírico muy utilizado más que sería interesante confirmar con el genotipaje de esos rebaños.

La colecta fue realizada desde la raíz de los mechones de lana, situados en la fosa paralumbar (Rojas et al., 2005), durante los meses de mayo y junio, tiempo suficiente para el trabajo de campo, además de coincidir con el inicio de inverno, época hasta la cual los ovinocultores mantenían la lana de los animales intacta, para enfrentar el clima litoráneo frío $\left(16^{\circ} \mathrm{C}\right)$ y húmedo (86\%) de Ite (Vizcarra, 2008). También se registró con un dinamómetro el peso vivo de las ovejas criollas y mestizas, buscando la mejor comprensión del biotipo de los grupos. Las CRICH eran más pesadas $(46,8 \mathrm{~kg})$, seguidamente las MHD $(45,7 \mathrm{~kg})$, en cuanto las CRIHU más leves y de menor tamaño $(38,8 \mathrm{~kg})$. Esta divergencia entre criollas puede ser debido a un linaje diferente, propio de cada ambiente. Otro motivo sería el incidente mestizaje de las CRICH con MHD, promovido por los agricultores para incrementar la aptitud cárnica en sus rebaños. Vale comentar que en los Humedales varios pastores Aymara realizan esas cruzas, pero la mayoría de mestizos no desarrollaban plenamente, por el ambiente hostil de ese ecosistema.

Las muestras de lana fueron embaladas individualmente en sobres carta (Figura 2), rotulados con sus respectivos datos y enviados desde Tacna (Perú), para análisis en el Laboratorio de Calidad de Lana, del Instituto de Estudios Indígenas de la Universidad Autónoma de Chiapas (UNACH), en San Cristóbal de Las Casas, Chiapas, México.

En el laboratorio cada muestra fue dividida en dos sub-muestras. La primera para análisis macroscópicas,

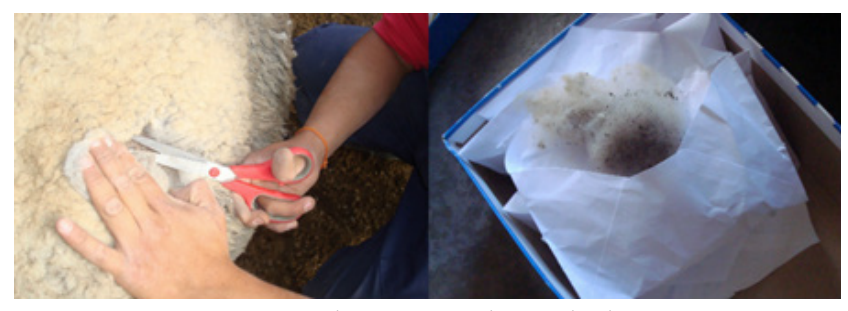

Figura 2. Muestreo de un mechón de lana y su envío postal para México (Sampling of a wool fleece and its posting to Mexico).

tales como determinación de proporciones (\%) de los tipos de fibra existentes en el mechón (largas-gruesas, cortas-finas y kemp), contando un número de 600, con lupa de aumento e iluminación. También se midió el largo de los tipos de fibra encontrados, con auxilio de una regla (Parés \& Perezgrovas, 2009).

La segunda sub-muestra fue usada en evaluaciones microscópicas de rendimiento post desengrase alcohólico y medición del diámetro de las fibras. Primero se colocó la lana en una balanza digital, registrando su peso sucio (PS), en seguida fue sumergida 24 horas a $21^{\circ} \mathrm{C}$, con alcohol isopropílico dentro de una jeringa de $60 \mathrm{cc}$, evitando presencia de burbujas de aire. Se enjuagó el mechón dos veces en isopropanol, con finalidad de retirar la grasa, después se exprimió de forma manual, para luego guardar en sobres de papel y secar por 24 horas a temperatura ambiente. Después la lana fue secada de forma controlada en incubadora, por 48 horas a $22^{\circ} \mathrm{C}$ y $63 \%$ de humedad. Esta sub-muestra fue pesada para obtener el peso limpio (PL), calculando con eso su rendimiento ( $\left.\mathrm{PL}^{*} 100 / \mathrm{PS}\right)$ después del desengrase alcohólico (García et al., 2005). Los datos macroscópicos y de rendimiento fueron registrados en planillas electrónicas.

De las 46 sub-muestras pesadas y limpias se escogieron aleatoriamente seis, pertenecientes a cuatro ovejas criollas de los Humedales y dos criollas de las chacras, por ser un número significativo para la medición microscópica del diámetro de fibra de esa etnia local, que está en peligro de extinción. Fueron realizados cortes transversales con bisturí (1 $\mathrm{mm})$, en la parte intermedia y basal de las fibras. La lana seccionada fue distribuida sobre una lámina con estiletes metálicos, depositando sobre ella varias gotas de resina epóxica para montaje permanente, siendo cubierta con lamínula, evitando burbujas de aire y después secada durante 10 días a temperatura ambiente. La lectura de los diámetros $(\mu)$ fue en 312 fibras por oveja, usando equipo de videomicromedición, microscopio óptico trinocular, cámara de video, monitor y micrómetro (Rojas et al., 2005).

Los datos macroscópicos y de rendimiento alcohólico de la lana se transcribieron a planillas electrónicas para su análisis usando el programa computacional R (R Development Core Team, 2011). Fue realizada estadística descriptiva (promedio y desvío estándar) de los valores encontrados en los tres grupos, para compararlos mediante análisis de varianza y Test Duncan a nivel de significancia del $5 \%$, con el paquete easyanova (Arnhold, 2013). Los diámetros de fibra fueron ordenados de menor a mayor en planillas electrónicas, 
para verificar el factor de confort (\% fibras $<30 \mu)$ y estructurar un histograma con la distribución de las frecuencias (Galdámez et al., 2012; Peña et al., 2016).

\section{RESULTADOS Y DISCUSIÓN}

\section{Características macroscópicas de LA LANA}

En lo referente a las características macroscópicas de la lana (Tabla I), la proporción de los tipos de fibra existentes no mostró largas-gruesas en las ovejas criollas, pero sí un porcentaje reducido en la lana de las mestizas HD (MHD). La mayoría de las fibras encontradas eran cortas-finas, tanto en las CRIHU, CRICH y MHD, por lo cual la punta de la mecha fue clasificada como cuadrada (Parés, 2009). Se sabe que en cuanto la fibra sea más fina, será más corta y en cuanto sea más gruesa, será más larga (Flores, 2008).

Estos resultados obtenidos de fibras cortas-finas son parecidos con los documentados por Perezgrovas et al. (2011) en el Merino español (99,6\%), así como los descritos por Parés et al. (2007a) en ovejas Assaf $(99,7 \%)$ de España o Baregesas $(99,9 \%)$ y Aura-Campan (100\%) de Francia, atribuyéndose la mayor presencia de ese tipo de fibra gracias al parentesco con el tronco Merino desde su formación (Parés et al. 2007b). Algo que también puede estar relacionado a las ovejas criollas de Ite, ya que en Perú los colonizadores españoles trajeron ovinos Merino (Flores et al., 2003). Un segundo motivo sería la "preferencia" de la mayoría de los criadores por ovinos de lana corta, como relataron en las visitas. Debido a que en verano, el calor litoráneo sofoca los animales, y sus dueños no saben o no poseen costumbre de trasquilarlos y procesar la lana, por lo cual intencionalmente fueron fijando esa característica a través de las generaciones, criterios tradicionales que son parte de la etnozootecnia (Alves et al., 2010). Algo semejante a la selección de criadores españoles de ovejas Aranesas, que evitaban animales con mechones largos, porque la lana colectaba materiales ásperos y suciedad en el pastoreo a campo (Parés et al., 2011a).

El último tipo de fibra registrado fueron las kemp (cortas gruesas), que en las ovejas CRIHU y CRICH eran porcentajes mínimos y próximos a los reportados por Galdámez et al. (2012), para la variedad gris de la oveja criolla Formosa en Argentina $(0,6 \%)$, y por García et al. (2005) en la variedad negra del Borrego mexicano Chiapas (0,7\%). En el caso de las ovejas MHD, el porcentaje de kemp fue mayor a las criollas y próximo del registrado por Galdámez et al. (2009) en ovejas criollas bolivianas (2,9\%). La poca cantidad de kemp de las criollas de Ite mostró superioridad de su lana a nivel industrial (Parés et al., 2011a), en comparación a las MHD y otras razas criollas con valores más elevados, como las ovejas estadounidenses Navajo-Churro (2 a $5 \%)$, Churras españolas $(8,1 \%)$ o las Manchegas negras $(9,6 \%)$, estudiadas por Sponenberg \& Taylor (2009), Rojas et al. (2005) y Perezgrovas et al. (2002), respectivamente. Se sabe que el kemp es un pelo corto y rígido, indeseado en la industria textil, por ser medulado y de diámetro muy grueso (> $80 \mu$ ), lo que disminuye la calidad y precio de la lana cuando es vendida (Amarilho-Silveira et al., 2015), ya que esa característica restringe la absorción de tintas para su coloración artificial, además de provocar fácil rompimiento de las fibras durante el proceso (Parés et al. 2011a).

Referente a la longitud de fibras (Tabla II), apenas las ovejas MHD presentaron largas-gruesas, con un tamaño mínimo. En el caso de las fibras cortas-finas, no hubo diferencia estadística significativa entre los tres grupos estudiados, registrando la mayor largura las criollas de los Humedales, con valor próximo al obtenido por ovejas criollas Tarahumara $(8,3 \mathrm{~cm})$ de México (Perea et al., 2008) y ovejas Junín ( $9 \mathrm{~cm}$ ), la única raza peruana registrada y productora de lana (Flores et al., 2007). Este mechón más largo puede ser por el clima frío y húmedo de los Humedales, sobre todo durante el inverno.

Las ovejas CRICH poseían fibras más cortas, casi semejantes en tamaño a las encontradas en las MHD, así como HD puras $(6,2 \mathrm{~cm})$ descritas por Ribeiro et al. (2015). Esto evidenciaría el mestizaje entre CRICH y MHD, que como se sabe es incentivado marcadamente por los agricultores. Parés et al. (2007a), Perezgrovas et al. (2011) y Peña et al. (2016) estudiaron diferentes razas de ovejas como el Merino Español $(3,3 \mathrm{~cm})$, la Aranesa (3,8 cm), Churra (3,9 cm), Latxa $(4 \mathrm{~cm})$ y Criolla Argentina $(4,3 \mathrm{~cm})$, las cuales poseían fibras cortas bien menores. Justificándose el Merino por su conocida aptitud para fibras cortas-finas y las otras razas por su mayor aptitud para carne o leche, sin selección para calidad de lana (Parés et al., 2011a). Situación que también ocurrió con las ovejas criollas y MHD de Ite, que eran criadas sólo para producir carne. Según Alencastre \& Gómez (2005), en Perú la carne es lo que más se aprovecha del ovino criollo, porque su producción lanar es escasa $(1,5 \mathrm{~kg})$, siendo que muchos dueños no trasquilan o realizan esto cada dos años.

Tabla I. Características macroscópicas de los tipos de fibra en lana de ovejas criollas y mestizas muestreadas en Ite, Perú (Macroscopic characteristics of fiber types on wool from creole and crossbred sheep sampled in Ite, Peru).

\begin{tabular}{lcccccc}
\hline Variables & \multicolumn{2}{c}{ CRIHU $(\mathrm{n}=20)$} & \multicolumn{2}{c}{ CRICH $(\mathrm{n}=20)$} & \multicolumn{2}{c}{ MHD $(\mathrm{n}=6)$} \\
\cline { 2 - 7 } & Promedio & DS & Promedio & DS & Promedio & DS \\
\hline Fibras largas-gruesas (\%) & - & - & - & - & 0,9 & 2,3 \\
Fibras cortas-finas (\%) & $99,5^{\mathrm{a}}$ & 0,3 & $99,6^{\mathrm{a}}$ & 0,3 & $96,4^{\mathrm{b}}$ & 0,5 \\
Fibras kemp (\%) & $0,5^{\mathrm{b}}$ & 0,1 & $0,4^{\mathrm{b}}$ & 0,1 & $2,7^{\mathrm{a}}$ & 0,3 \\
\hline
\end{tabular}

Promedios con letra diferente $(a, b)$ no son iguales por el Test Duncan al $5 \%$

$\mathrm{CRIHU}=$ Criollas de los Humedales; $\mathrm{CRICH}=$ Criollas de las chacras; $\mathrm{MHD}=$ Mestizas Hampshire Down; $\mathrm{n}$ = número; DS = Desvío Estándar 
Tabla II. Características macroscópicas de longitud de lana de ovejas criollas y mestizas muestreadas en Ite, Perú (Macroscopic characteristics of wool length from creole and crossbred sheep sampled in lte, Peru).

\begin{tabular}{lcccccc}
\hline Variables & \multicolumn{2}{c}{ CRIHU $(\mathrm{n}=20)$} & \multicolumn{2}{c}{ CRICH $(\mathrm{n}=20)$} & \multicolumn{2}{c}{ MHD $(\mathrm{n}=6)$} \\
\cline { 2 - 7 } & Promedio & DS & Promedio & DS & Promedio & DS \\
\hline Fibras largas-gruesas $(\mathrm{cm})$ & - & - & - & - & 1,1 & 2,8 \\
Fibras cortas-finas $(\mathrm{cm})$ & $8,6^{\mathrm{a}}$ & 0,7 & $6,8^{\mathrm{a}}$ & 0,7 & $6,4^{\mathrm{a}}$ & 1,2 \\
Fibras kemp (cm) & $0,4^{\mathrm{a}}$ & 0,1 & $0,5^{\mathrm{a}}$ & 0,1 & $0,6^{\mathrm{a}}$ & 0,2 \\
\hline
\end{tabular}

Promedios con letra diferente $(a, b)$ no son iguales por el Test Duncan al $5 \%$

$\mathrm{CRIHU}=$ Criollas de los Humedales; $\mathrm{CRICH}=$ Criollas de las chacras; $\mathrm{MHD}=$ Mestizas Hampshire Down; $\mathrm{n}=$ número; DS = Desvío Estándar; $\mathrm{cm}=$ centímetros

La temperatura más calurosa de las chacras puede haber influenciado naturalmente en las criollas a través de las décadas, y también la selección artificial de los criadores, para un menor tamaño de mechón. Se sabe que ese ambiente posee topografía más alta, con 175 metros sobre el nivel del mar (MDI, 2014b), provocando menos humedad y mayor exposición al sol. Para Galdámez et al. (2009) la diferencia de ambientes influyó en el tamaño de fibra de ovejas criollas bolivianas, donde las que vivían en los valles mostraron $8,2 \mathrm{~cm}$ de largura, y otras criadas en la cordillera por el frío tenían mechones más largos con 12,1 cm.

La largura de las fibras de los tres grupos evaluados (CRIHU, CRICH e MHD) fue superior a $5 \mathrm{~cm}$, lo que demuestra aptitud para el sistema de peinado (tejidos finos). Si las fibras fuesen menores a los $5 \mathrm{~cm}$ serían destinadas para cardado. Esto indica la importancia de esa característica física, para decidir el sistema de transformación textil a usar (Guzmán \& Aliaga, 2010), así como para programas de selección y comercialización de fibras (Flores, 2008). Según la clasificación de Parés (2009), los porcentajes de los tipos de fibra encontrados, más el formato del mechón y largura, clasificarían la lana de las ovejas criollas de Ite como "entrefina-fina" y de las MHD como "entrefina-ordinaria", mostrando superioridad los animales criollos, lo que podría ser explotado paralelamente a la producción cárnica, comercializando esta materia prima en la industria textil mecanizada.

En el caso de seleccionar ovinos con fibras más largas, como es el caso de las ovejas CRIHU, podría ocurrir lo mismo que con el Borrego criollo Chiapas, el cual en 1995 tenía fibras de 8,3 cm de longitud, antes de ser seleccionado bajo criterios tradicionales de las pastoras Tzotziles e investigadores, obteniendo mechones más largos $(11,4 \mathrm{~cm})$ después de ocho años de trabajo (García et al., 2005). Hecho que diversificaría la ovinocultura de Ite, pues esas fibras son adecuadas en la confección textil artesanal, generando más una fuente de renta económica para los pastores Aymara y también la conservación de los ovinos locales, como está sucediendo en comunidades pastoriles de Bolivia, donde el gobierno apoya su producción con maquinaria y capacitación para que puedan competir en el mercado de lanas (Stemmer et al., 2017). Se sabe que la elaboración artesanal de tejidos en los Andes es una tradición realizada por los indígenas desde hace siglos, utilizando fibras de camélidos sudamericanos (Monzón et al., 2012). La producción textil artesanal mejora la economía de los criadores, promueve integración social en la comunidad y mantiene la diversidad genética de los ovinos criollos, por la búsqueda de lana colorida para confección de ropas típicas (Peña et al., 2016).

En lo referente a la largura de las fibras kemp, fue muy corta en los tres grupos estudiados, con resultados próximos a la oveja Baregesa $(0,7 \mathrm{~cm})$ reportada por Parés et al. (2007a). No en tanto, contrario a los valores obtenidos por ovejas criollas Tarahumara $(4,4 \mathrm{~cm})$ y Chiapas (4,6 cm) en México (Perea et al., 2008).

\section{Características microscópicas de la laNA}

Las características de relevancia industrial de la lana son el rendimiento post desengrase alcohólico y las fibras finas. Debiendo poseer menos de $10 \%$ de grasa para proceder a un tratamiento industrial menos oneroso (Tinoco, 2009). En el análisis microscópico del rendimiento post lavado alcohólico de las 46 muestras de lana (Tabla III), el grupo CRIHU (78,9\%) fue mejor a los otros por la menor presencia de suarda (grasa) en las fibras. Algo que según Parés et al. (2011a) y Parés et al. (2011b) es típico de las razas que habitan en climas fríos, como el ambiente de los Humedales, ya que existe poca sudoración en el cuerpo del animal, produciendo menos suarda, lo que visualmente muestra en los ovinos unos mechones semi-abiertos (no compactos).

Tabla III. Característica microscópica de rendimiento alcohólico de lana de ovejas criollas y mestizas muestreadas en Ite, Perú (Microscopic characteristic of alcoholic yield of wool from creole and crossbred sheep sampled in Ite, Peru).

\begin{tabular}{lcccccc}
\hline Variables & \multicolumn{2}{c}{ CRIHU $(\mathrm{n}=20)$} & \multicolumn{2}{c}{ CRICH $(\mathrm{n}=20)$} & \multicolumn{2}{c}{ MHD $(\mathrm{n}=6)$} \\
\cline { 2 - 7 } & Promedio & DS & Promedio & DS & Promedio & DS \\
\hline Rendimiento post lavado (\%) & $78,9^{\text {a }}$ & 2,3 & $67,8^{\text {b }}$ & 2,3 & $66,6^{\text {b }}$ & 4,1 \\
\hline
\end{tabular}

Promedios con letra diferente (a, b) no son iguales por el Test Duncan al $5 \%$

CRIHU = Criollas de los Humedales; CRICH = Criollas de las chacras; MHD = Mestizas Hampshire Down; n = número; DS = Desvío Estándar 
El valor registrado por las CRIHU fue próximo al estudio de ovejas Manchegas negras, con 77,8\% (Perezgrovas et al., 2002) y Xisqueta, con $80,9 \%$ (Parés \& Perezgrovas, 2009), no en tanto superior a los resultados de otras ovejas criollas americanas, como la Tarahumara (73,2\%) de México (Perea et al., 2008), NavajoChurro (67 a 72\%) de los Estados Unidos (Sponenberg \& Taylor, 2009), y criollas bolivianas $(64,2 \%)$ en los Andes (Galdámez et al., 2009). García et al. (2005) indicaron que en 1995, el rendimiento al post desengrase alcohólico de la lana del Borrego Chiapas era 81,5\%, y después de ocho años de selección mejoró para 83,1\%.

Las CRICH registraron menor rendimiento que las CRIHU al post desengrase alcohólico, obteniendo porcentajes próximos a las MHD, lo que podría confirmar nuevamente su parentesco. Vale comentar como valor de referencia, el rendimiento de $61,8 \%$ obtenido en ovejas HD puras, estudiadas por Ribeiro et al. (2015). El bajo rendimiento de las CRICH también puede ser debido a ciertas características todavía vigentes del Merino español, que en el estudio de Perezgrovas et al. (2011) mostró inferioridad post lavado (53\%), algo típico de la lana de esa raza. Parés et al. (2007b) relataron que cuanto más cortas son las fibras, tendrán más bajos los rendimientos alcohólicos, por la mayor presencia de "suarda", que protege de agentes externos del medio ambiente, así como lubrifica las fibras para mantener su flexibilidad y elasticidad (Flores, 2008), dando apariencia de una lana cerrada (aglutinada) y algo amarilla (Parés et al., 2011b). Peculiaridades encontradas en los muestreos a campo de lana, en algunas ovejas CRICH y sobre todo en las MHD, observando muchas veces una cera entre sus mechas. Se sabe que el clima caluroso de las chacras provoca que el ovino sude más para regular su temperatura corporal, lo que fomenta la presencia de suarda (Elvira, 2009).

Conforme evaluación microscópica del diámetro de las fibras, el promedio de las seis ovejas criollas de Ite fue $24 \mu$. Valor que para Díaz (2007) corresponde a fibras medianamente finas (20-26 $\mu)$, buscadas por el mercado chino, ya que las finas y superfinas $(<18$ $\mu)$ son para otro mercado internacional más exigente, como el europeo y estadounidense. Según Mueller (1999) las lanas finas poseen de 19 a $21 \mu$, en cuanto las superfinas menos de $19 \mu$ y ultrafinas menos de $16 \mu$. En la clasificación española la lana de los ovinos de Ite era entrefina de calidad media, por estar en el intervalo de 24 a $36 \mu$ (Parés et al., 2011b). Guzmán \& Aliaga (2010) relataron que el diámetro de fibra es la característica física más importante, bajo el punto de vista comercial, porque determina el precio y uso en el procesamiento industrial, siendo la puntuación del diámetro encontrado AAA (23,5 a 24,5 $\mu)$, de acuerdo con el sistema peruano de clasificación de lanas.

Las ovejas criollas de Ite $(24 \mu)$ obtuvieron una finura de fibra próxima a ovejas criollas $(24,8 \mu)$ del altiplano sur peruano (Gómez \& Gómez, 2009), criollas de la sierra $(25,1 \mu)$ y valles $(24,5 \mu)$ bolivianos (Galdámez et al., 2009), así como a la raza Junín, 23 a $25 \mu$ (Flores et al., 2007). Inclusive no tan distantes de Merinos de la Patagonia, con 22,8 $\mu$ (Frey et al., 2009) y superiores a algunas razas especializadas como la Ideal, 23,6 $\mu$ (Franz et al., 2008) o la Corriedale, 26,1 $\mu$ (Guzmán \&
Aliaga, 2010). Además de eso, superior también a otras razas criollas latinoamericanas, como las ovejas argentinas Formosa, que poseían entre 27 y $36 \mu$ (Galdámez et al., 2012), el borrego mexicano Chiapas, variedad negra, con 48,1 $\mu$ (Perezgrovas et al., 2002), o razas nativas de España y Francia estudiadas por Parés et al. (2007a), destacando la Aranesa $(29,5 \mu)$, Baregesa $(32 \mu)$ y Castillonesa $(31,7 \mu)$. El sexo influencia la finura de la fibra, pues las ovejas tienen menor diámetro que los carneros. La edad impacta, siendo la lana de animales jóvenes y viejos más fina. También el frío y el hambre disminuyen el diámetro de las fibras (Mendives, 2007; Flores, 2008). En este caso, la espesura intermedia de la fibra de la lana de las ovejas criollas de Ite, además de los factores mencionados tendría influencia de los troncos ancestrales que con mayor frecuencia llegaron a Perú, como son el Churro, Merino y Lacha, que poseen lana gruesa, fina y entrefina, respectivamente (Flores et al., 2003).

Referente al "factor de confort" (diámetro $\leq 30 \mu$ ), de las 1872 fibras analizadas en las seis ovejas criollas de Ite, $88,8 \%$ de estas presentaban esa especial característica y apenas $11,2 \%$ de las fibras tenían diámetros gruesos, los cuales son indeseados, pues provocan poca flexibilidad de los extremos, causando prurito en la piel humana (Sacchero, 2005; Peña et al., 2016). El hecho de que los ovinos criollos de Ite tienen fibras que proveen confort, posibilita el acceso a lana de alta calidad, obteniendo mejor precio en su venta y de sus subproductos (Franz et al., 2008). Estos resultados superaron los obtenidos por Peña et al. (2016) en cuatro poblaciones de ovinos criollos argentinos, donde el mejor resultado fue $78,8 \%$ del rebaño de la provincia de Salta, en cuanto el rebaño de Corrientes alcanzó 34\%, mostrando lana gruesa y poco confortable.

Las frecuencias del histograma indicaron abundancia de fibras finas-cortas, concentradas entre 16 a 32 $\mu$, que era 89,4\% de las 1872 fibras analizadas (Figura 3). Esto mostró mayor porcentaje de mechones cortos, estructurando una monocapa (Parés \& Perezgrovas,

\section{Diâmetros das fibras de ovelhas crioulas de Ite}

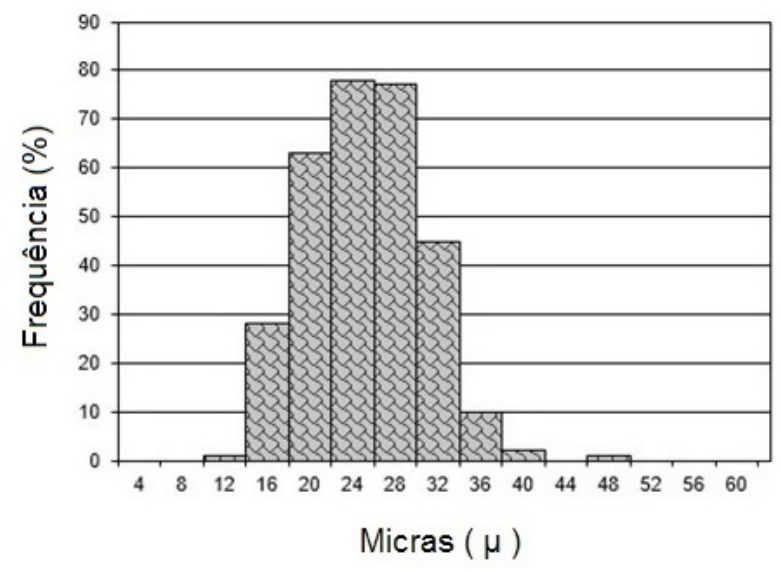

Figura 3. Histograma de distribución de los diámetros de fibra en ovejas criollas de Ite, Perú (Histogram of distribution of fiber diameters in creole sheep from Ite, Peru). 
2009), lo que podría evidenciar una oveja criolla cruzada con razas de fibra corta, como los híbridos de HD presentes en la localidad, o la falta de selección para producción de lana (Rojas et al., 2005). Pues los ovinos primitivos tienen doble capa, como la lana de ovejas Navajo-Churro (Sponenberg \& Taylor, 2009) y del Borrego Chiapas (Perezgrovas et al., 2002), que presentan dos tipos definidos de fibras, las cortas-finas y largasgruesas, característica peculiar que puede deberse al aislamiento geográfico y ausencia de mestizaje con otras razas (Perezgrovas, 1998).

En Perú, la lana es obtenida mediante trasquila de los ovinos vivos o retirándola de las pieles después de beneficiar el animal, siendo llamados de pomallos las pieles con lanas cortas y de enlanados las pieles con lanas largas (Díaz, 2013). Después de eso la lana puede ser destinada para uso industrial, donde el valor está determinado por el diámetro de la fibra y el color blanco; o en el uso artesanal, que busca fibras largas y gruesas, así como diversidad de colores (Galdámez et al., 2012). El mayor uso de la lana es en la confección artesanal de ropas indígenas (Tinoco, 2009), reconocidas por el turismo nacional e internacional, ya que eso representa la combinación de cultura, técnica y recurso genético animal (Sponenberg \& Taylor, 2009). En el uso industrial existen algunas empresas peruanas que adquieren lana ovina, para transformación y comercialización de productos semi-procesados, hilos o tejidos. El empresario textil encuentra en la lana ovina una alternativa para mantener ocupada la maquinaria, cuando no posee suficiente fibra de alpaca, pudiendo ser mezclada con esta o con algodón. Es importante indicar que no existen ovinocultores organizados, ni entidades estatales preocupadas, diferente a la realidad de los criadores de alpacas, que poseen un mercado estructurado y un mayor precio por la fibra que producen (Gómez \& Gómez, 2009; Tinoco, 2009).

\section{CONCLUSIONES}

Las ovejas criollas de las chacras y las mestizas Hampshire Down mostraron una calidad de lana adecuada para la confección textil industrial, por sus características macroscópicas y de rendimiento alcohólico que fueron próximas, confirmando el mestizaje étnico entre esos dos grupos, como fue mencionado por los agricultores. Sin embargo, la lana de las ovejas criollas de los Humedales registró superioridad, además de tendencia hacia la confección textil artesanal, lo que podría ser otra fuente de renta económica para los pastores Aymara que sólo producen carne ovina. En general, la finura de la fibra de lana de las ovejas criollas de Ite fue muy buena, compitiendo inclusive con razas especializadas en producción lanar, ya que puede brindar confort en las ropas que sean producidas con ese material, así como facilidad en la tinción y otros procesos industriales. Es urgente crear núcleos de ovinos locales, buscando su conservación y fomento entre los criadores, quienes deben organizarse y pedir apoyo gubernamental para la confección textil, logrando de esa manera una ovinocultura sustentable en Ite.

\section{BIBLIOGRAFÍA}

Agrodata Perú 2018, 'Lana sin cardar Perú exportación 2018 Enero', acceso 01 Mayo 2018, https://www.agrodataperu.com/2018/02/ lana-sin-cardar-peru-exportacion-2018-enero.html

Alencastre, RD \& Gómez, NU 2005, 'Comportamiento reproductivo del ovino criollo en el altiplano peruano', Archivos de Zootecnia, vol. 54, no. 206-207, pp. 541-544.

Aliaga, JLG 2009, 'Posibilidades del desarrollo de la crianza ovina en el Perú', en Memorias III Foro Regional sobre Ovinos Criollos, Perú, Cusco, acceso 17 Noviembre 2015, http://www.arariwa.org. pe/8posibilidades.pdf

Alves, AGC, Pires, DAF \& Ribeiro, MN 2010, 'Conhecimento local e produção animal: uma perspectiva baseada na etnozootecnia', Archivos de Zootecnia, vol. 59 (R), pp. 45-56.

Amarilho-Silveira, F, Brondani, WC \& Lemes, JS 2015, 'Lã: características e fatores de produção', Archivos de Zootecnia, vol. 64 (R), pp. 13-24.

Arnhold, E 2013, 'Package in the R environment for analysis of variance and complementary analyses', Brazilian Journal of Veterinary Research and Animal Science, vol. 50, no. 6, pp. 488-492.

De la Barra, R, Carvajal, A, Uribe, H, Martínez, ME, Gonzalo, C, Arranz, J \& San Primitivo, F 2011 , 'El ovino criollo Chilote y su potencial productivo', Animal Genetic Resources, vol. 48, pp. 93-99.

Díaz, RIR 2007, 'Sector ovinos en el Perú con perspectivas al 2015', en Memorias V Congreso Latinoamericano de Especialistas en Pequeños Rumiantes y Camélidos Sudamericanos, Argentina, Mendoza, acceso 27 Julio 2014, http://www.produccion-animal.com. ar/produccion_ovina/produccion_ovina/106-diaz_ovinos_peru.pdf

Díaz, RIR 2013, 'Cadena productiva de ovinos', Ministerio de Agricultura y Riego, Lima, acceso 20 Septiembre 2014, http://agroaldia. minag.gob.pe/biblioteca/download/pdf/agroeconomia/agroeconomia_ovino.pdf

Elvira, MG 2009, 'El ovino: La fábrica biológica de lana', Carpeta Técnica EEA Esquel, no. 32, pp. 147-150.

Flores, ER, Cruz, JA \& López, M 2003, 'Manejo y uso de los recursos genéticos en los sistemas de producción ovina de los Andes Centrales del Perú', en Memorias I Taller sobre uso y manejo de los recursos genéticos de rumiantes menores en sistemas ganaderos tradicionales en países de Sudamérica, Argentina, Bariloche, acceso 12 Mayo 2014, http://www.produccion-animal.com.ar/produccion_de_camelidos/ camelidos_general/99-recursos_geneticos.pdf

Flores, ER, Cruz, JA \& López, M 2007, 'Management of sheep genetic resources in the central Andes of Peru', en Tempelman, KA \& Cardellino, RA, People and animals. Traditional livestock keepers: guardians of domestic animal diversity, Food and Agriculture Organization of the United Nations, Roma, acceso 03 Marzo 2014, http://www.fao. org/3/a-a1057e/a1057e02.pdf

Flores, DS 2008, 'Crianza de ovinos', Articulo.org, acceso 01 Abril 2013, http://www.articulo.org/articulo/3625/crianza_de_ovinos_i.html

Franz, N, Aguilar, D, Pourrain, A \& Robson, C 2008, 'Una alternativa para la producción ovina: las lanas finas', Noticias y Comentarios - Estación Experimental Agropecuaria Mercedes, no. 439, pp. 1-3.

Frey, A, Martín, N, De Caro, A, Álvarez, DU \& Elvira, M 2009, 'Variación del diámetro promedio de fibras de lana en ovejas Merino trasladadas desde la Patagonia a la región pampeana', Archivos Latinoamericanos de Producción Animal, vol. 17, no. 1-2, pp. 9-14.

Fulcrand, BT 2005, 'Melhorando o desempenho de raças locais de ovelhas', Agriculturas, vol. 2, no. 4, pp. 16-19.

Galdámez, CF, Perezgrovas, RG, Galarza, AB, Galdámez, DF \& Stemmer, A 2009, 'Análisis de la mecha y la fibra de lana en las ovejas criollas nativas de dos zonas ecológicas de Bolivia', en Memorias VI Congreso Latinoamericano de Especialistas en Pequeños Rumiantes y Camélidos Sudamericanos, México, Querétaro, acceso 28 Enero 2016, http://www.aleprycs.net/documents/21709/27370/ PRODUCCION+LANA.pdf

Galdámez, D, De La Rosa, S, Perezgrovas, R, Revidatti, MA \& Rodríguez, G 2012, 'Características macroscópicas y microscópicas de la mecha y la fibra de lana en la oveja autóctona Formosa de Argentina', Actas Iberoamericanas de Conservación Animal, vol. 2, pp. 309-312. 
García, VG, Perezgrovas, RG, Castro, HG, Zaragoza, LM \& Rojas, ALC 2005, 'Impacto de la selección inducida sobre características de la fibra en las razas de ganado lanar de Chiapas', Archivos de Zootecnia, vol. 54, no. 206-207, pp. 289-294.

Gómez, NCU \& Gómez, JWU 2009, 'Importancia del recurso ovino peruano en el desarrollo rural sostenible', en Delgado, JVB \& Nogales, SB, Biodiversidad ovina Iberoamericana. Caracterización y uso sustentable, Universidad de Córdoba, Córdoba, acceso 25 Agosto 2015, http://www.uco.es/conbiand/pdf/biodiversidad_ovina.pdf

Guzmán, JCB \& Aliaga, JLG 2010, 'Evaluación del método de clasificación del vellón en ovino Corriedale (Ovis aries) en la SAIS Pachacútec', Sitio Argentino de Producción Animal, acceso 14 Mayo 2015, http://www.produccion-animal.com.ar/produccion_ovina/ produccion_ovina_lana/19-vellon_ovino.pdf

Mendives, JAA 2007, 'Importancia de los ovinos tropicales introducidos al país: características productivas y reproductivas', Archivos Latinoamericanos de Producción Animal, vol. 15, spl. 1, pp. 310-315.

Montesinos, IS, Catachura, A, Sánchez, J, Franco, JL, Arnhold, E, McManus, C, Fioravanti, MCS \& Sereno, JRB 2015, 'Caracterización de ovinos en el litoral sur del Perú', Animal Genetic Resources, vol. 56 , pp. 55-62.

Monzón, M, Lanari, MR, López, S, Zubizarreta, JL \& Subiabre, M 2012, 'Caracterización de sistemas ovinos en Patagonia', en Memorias XVI Jornadas Nacionales de Extensión Rural y VIII del Mercosur, Argentina, Concordia, acceso 08 Enero 2106, http://inta.gob.ar/ documentos/caracterizacion-de-sistemas-ovinos-criollos

Mueller, J 1999, 'Producción de lana superfina', en Memorias II Congreso Lanero Argentino, Argentina, Trelew, acceso 28 Marzo 2015, http://inta.gob.ar/sites/default/files/script-tmp-inta-produccion_de_lana_superfina.pdf

Municipalidad Distrital de Ite (MDI), 2014a, 'Censo poblacional de rumiantes menores del distrito de Ite', Proyecto Mejoramiento de capacidades en buenas prácticas pecuarias, para el tratamiento del ganado ovino.

Municipalidad Distrital de lte (MDI), 2014b, 'Plan de desarrollo concertado distrital al 2021', acceso 13 Diciembre 2015, http://www. muniite.gob.pe/transparencia/planiamiento-y-organizacion/ PDC-CAPITULO-I-II-III.pdf

Parés, PM 2009, 'Expresiones etnológicas para la lana entrefina', Pequeños Rumiantes, vol. 10, no. 2, pp. 27-31.

Parés, PM, Perezgrovas, R \& Jordana, J 2007a, 'Análisis comparativo del vellón en diferentes razas ovinas españolas y francesas' en Memorias VIII Simposio Iberoamericano sobre Conservación y Utilización de Recursos Zoogenéticos, Ecuador, Quevedo, acceso 06 Enero 2016, http://www.rac.uab.es/bibliografia/articles/ Aranesa/CYTED2007.pdf

Parés, PM, Perezgrovas, RG \& Jordana, J 2007b, 'La raza ovina de Aura-Campan: un recuerdo de merinización en el Pirineo central', Redvet, vol. 8, no. 10, pp. 1-11.

Parés, PM \& Perezgrovas, R 2009, 'Análisis de la mecha y las fibras de lana en la raza ovina Xisqueta', Revista de la Facultad de Ciencias Veterinarias, vol. 50, n. 1, pp. 53-58.

Parés, PM, Jordana, J \& Perezgrovas, R 2011 a, 'Study of wool characteristics in the Aranese ovine breed', International Journal of Morphology, vol. 29, no. 1, pp. 123-126.

Parés, PM, Perezgrovas, R \& Jordana, J 2011 b, 'Comparison of four European endangered red sheep based on fleece characteristics', Animal Genetic Resources, vol. 48, pp. 85-91.

Peña, S, López, GA, Abbiati, NN, Género, ER \& Martínez, RD 2015, 'Avances en la caracterización zootécnica de la raza Criolla Argentina', Revista de Divulgación Técnica Agropecuaria, Agroindustrial y Ambiental, vol. 2, no. 1, pp. 11-20.

Peña, S, Sacchero, D, Maurino, J, López, GA, Abbiati, NN, Género, ER \& Martínez, RD 2016, 'Caracterización de la lana de ovejas Criollas argentinas en cuatro ambientes diferentes', Archivos de Zootecnia, vol. 65, no. 249, pp. 13-19.

Perea, T, Perezgrovas, R, Rodriguez, G, Jaramillo, E, Rubio, E \& Zaragoza, L 2008, 'Características del vellón y la mecha de lana en la oveja Autóctona de la sierra Tarahumara, Chihuahua, México', en Memorias IX Simposio Iberoamericano sobre Conservación y Utilización de Recursos Zoogenéticos, Argentina, Buenos Aires, acceso 27 Marzo 2016, http://www.uco.es/conbiand/pdf/argentina2008.pdf

Perezgrovas, RG 1998, 'Comparación de recursos genéticos: el Borrego Chiapas (México) y las razas autóctonas de origen español', Archivos de Zootecnia, vol. 47, no. 178-179, pp. 425-430.

Perezgrovas, RG \& Castro, HG 2000, 'El borrego Chiapas y el sistema tradicional de manejo de ovinos entre las pastoras Tzotziles', Archivos de Zootecnia, vol. 49, no. 187, pp. 391-403.

Perezgrovas, RG, Zaragoza, LM, Rodríguez, GG, Lozano, J, Bouzada, JAR \& Pérez, SEM 2002, 'Comparación de la mecha de lana en ovinos del tronco ibérico: el Borrego Chiapas negro (México) y la oveja Manchega española', en Memorias V Congreso de la Sociedad Española para los Recursos Genéticos Animales y III Congreso Ibérico sobre Recursos Genéticos Animales, España, Madrid, acceso 23 Octubre 2015, https://www.researchgate.net/ publication/257934890_Comparacion_de_la_mecha_de_lana_ en_ovinos_del_tronco_iberico_el_borrego_Chiapas_negro_Mexico_y_la_oveja_Manchega_espanola

Perezgrovas, R, Parés, PM, Hummel, J, Zaragoza, L \& Delgado, JV 2011 , 'Características de la lana en las ovejas autóctonas Ibicenca, Merino, Merino de Grazalema (España) y Merino Socorro (México)', Actas Iberoamericanas de Conservación Animal, vol. 1, pp. 380-383.

R Development Core Team R: A language and environment for statistical computing 2011, R Foundation for Statistical Computing, Vienna, Austria, http://www.Rproject.org

Ribeiro, ELA, Pires, CC, Mizubuti, IY, Silva, LDF, Castro, FABC, Koritiaki, NA, Pereira, ES \& Pinto, AP 2015, 'Characteristics of the wool produced by ewes raised for meat production', Revista Brasileira de Zootecnia, vol. 44, no. 11, pp. 405-411.

Rojas, ALC, Perezgrovas, RG, Rodríguez, GG, Russo-Almeida, P \& Anzola Vásquez, H 2005, 'Caracterización macro y microscópica de la lana en ovinos autóctonos iberoamericanos de vellón blanco', Archivos de Zootecnia, vol. 54, no. 206-207, pp. 477-483.

Sacchero, D 2005, 'Utilización de medidas objetivas para determinar calidad en lanas', en Memorias VII Curso de Actualización Ovina, Argentina, Bariloche, acceso 18 Septiembre 2015, http://www.minagri. gob.ar/site/ganaderia/ovinos/05=Documentaci\%C3\%B3n\%20 Tecnica/01-Lanas/_archivos/000000_Utilizacion\%20de\%20 medidas $\% 20$ objetivas $\% 20$ para $\% 20$ determinar $\% 20$ calidad $\% 20$ de\%20lana.pdf

Sponenberg, DP \& Taylor, C 2009, Navajo-Churro sheep and wool in the United States', Animal Genetic Resources, vol. 45, pp. 99-105.

Stemmer, A, Caballero, T, Zambrana, L \& Robalino, T 2017, 'Adaptación tecnológica para transformación de fibras de razas locales: cardadoras y ruecas eléctricas', Actas Iberoamericanas de Conservación Animal, vol. 10, pp. 19-26.

Tinoco, OG 2009, 'Cadena productiva de lana de oveja en el sector textil y de confecciones', Industrial data, vol. 12, no. 2, pp. 73-80.

Viana, JGA 2008, 'Governança da cadeia produtiva da ovinocultura no Rio Grande do Sul: Estudo de caso à luz dos custos de transação e produção', Dissertação, Universidade Federal de Santa Maria, acesso 25 Agosto 2015, http://cascavel.ufsm.br/tede//tde_busca/ arquivo. php? codArquivo $=1756$

Vizcarra, JK 2008, 'Los Humedales de Ite: Un potencial ecoturístico', acceso 14 Abril 2012, http://www.turismotacna.com/LIBRO_HUMEDALES_DE_ITE_OGDTURTACNA.pdf 